\title{
100 Jahre Hautklinik Ludwigshafen: Mit Blick nach vorne
}

\author{
100 Years of Skin Hospital Ludwigshafen: Looking Ahead
}

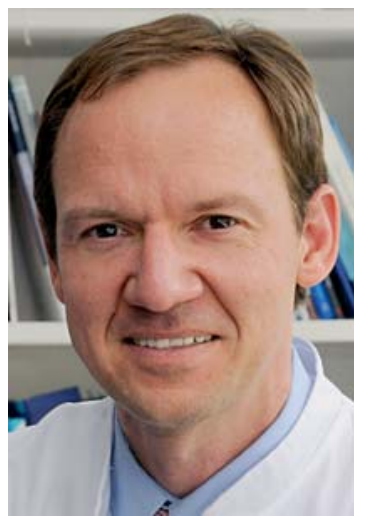

Prof. Dr. med. Edgar Dippel
Bibliografie

DOI http://dx.doi.org/

10.1055/s-0030-1256629

Akt Dermatol 2011; 37; 275

(c) Georg Thieme Verlag KG .

Stuttgart · New York

ISSN 0340-2541

Korrespondenzadresse

Prof. Dr. med. Edgar Dippel

Hautklinik/Hauttumorzentrum

Rheinpfalz

Klinikum Ludwigshafen

Bremserstraße 79

67063 Ludwigshafen

dippele@klilu.de
Die Hautklinik Ludwigshafen blickt auf eine 100jährige Geschichte zurück. Dr. Siegfried Fuß begründete die Hautklinik am Städtischen Klinikum im Dezember 1910. Seine dermatologische Ausbildung absolvierte er an der Hautklinik am Rudolf-Virchow-Krankenhaus in Berlin unter Abraham Buschke. Mit einem Schmunzeln erinnere ich mich gerne daran, dass auch ich 90 Jahre später meine dermatologische Ausbildung in denselben Räumlichkeiten begann.

In Ludwigshafen war schon zu Beginn der Jahrhundertwende eine enge Bindung zur Badischen Anilin- und Sodafabrik (BASF) dokumentiert. Die meisten der stationären Patienten litten an infektiösen Erkrankungen wie der Scabies, der Malaria und an Geschlechtskrankheiten, aber auch schon damals wurden regelmäßig operative Eingriffe durchgeführt. Dr. Siegfried Fuß übergab nach 40-jähriger Dienstzeit eine Klinik mit derzeit über 100 Betten an den Doz. Dr. Werner Schmidt, der 8 Jahre später die städtische Hautklinik Mannheim übernahm. Nachfolger wurde 1958 Prof. Dr. Paul Zierz, dessen besonderer Verdienst die Einrichtung von Laboren zur speziellen Diagnostik in der Allergologie, Andrologie und Mykologie waren. Nach dem frühen Tod von Prof. Zierz wurde Prof. Dr. Georg Brehm 1972 Direktor der Hautklinik. Er erreichte die Einrichtung eines histologischen und immunhistologischen Labors, das bis heute ein wichtiger Bestandteil Hautklinik ist. Zusammen mit Prof. Dr. Hansotto Zaun und Prof. Dr. Hans-Joachim Heite gegründete Prof. Dr. Brehm 1975 die Zeitschrift „Aktuelle Dermatologie“. Nach seiner Versetzung in den Ruhestand übernahm Prof. Dr. Volker Voigtländer die Leitung der Klinik. Nach neuer klinischer Ausrichtung mit operativem Schwerpunkt konnte Prof. Dr. Voigtländer die Auslastung der Klinik zunehmend erhöhen. Dabei wurden im Jahr 2007 bei 45 stationären Betten bis zu 1800 Patienten stationär behandelt. Die gute und kollegiale Zusammenarbeit mit den niedergelassenen Fachkollegen war dabei ein wichtiger Pfeiler dieser Erfolgsgeschichte. Im Februar 2008 fiel mir die Ehre zu, seine Nachfolge anzutreten.
Angesichts der zunehmenden Häufigkeit von Hautkrebserkrankungen in der Bevölkerung von Rheinland-Pfalz bedarf es weiterer strukturierter Anstrengungen, um dem Problem Hautkrebs zukünftig umfassend entgegentreten zu können. Ein wichtiger Schritt war die Etablierung eines Hauttumorzentrums mit interdisziplinären Vernetzung und regelmäßiger interdisziplinärer Tumorkonferenz. Am 7 7. 2009 wurde das Hauttumorzentrum Rheinpfalz am Klinikum Ludwigshafen erfolgreich von OnkoZert zertifiziert. Dabei wurde die onkologische Versorgungsstruktur durch die Implementierung der dermatologischen Onkologie, der klinischen Forschung mit dem frühen Zugang zu neuen wirksamen onkologischen Therapeutika und nicht zuletzt durch psychologische und soziale Betreuung der Patienten entscheidend erweitert. Die Interdisziplinarität der Organzentren stellt mit den unterschiedlichen Blickwinkeln der einzelnen Fachgruppen die individuelle klinische Situation des Patienten mehr in den Mittelpunkt der diagnostischen und therapeutischen Entscheidungen und Empfehlungen. Dieser Weg zur individualisierten onkologischen Therapie wird begleitet von nationalen und internationalen Leitlinienempfehlungen im Entscheidungsprozess. Die Hautklinik hat mit dieser Entwicklung einen weiteren Wachstumsprozess erlebt. Die Anzahl der stationären Betten stieg auf 48 und die variable Belegung von 6 weiteren Betten führte zur Behandlung von derzeit jährlich 2450 stationären Patienten. Der Blick ist weiter nach vorne gerichtet. Der Ausbau immunologischer Therapien bei onkologischen und entzündlichen Therapien sowie die Entwicklung von weiteren palliativen Versorgungsstrukturen sind neue Ziele für die nächste Dekade der Hautklinik.

Mit besten Grüßen aus Ludwigshafen am Rhein,

Ihr

Prof. Dr. Edgar Dippel 